\title{
Graph of brain structures grading for early detection of Alzheimer's disease
}

\author{
Kilian Hett ${ }^{1,2}$, Vinh-Thong Ta ${ }^{1,2,3}$, José V. Manjón ${ }^{4}$, and Pierrick Coupé ${ }^{1,2}$ \\ and the Alzheimer's Disease Neuroimaging Initiative ${ }^{\star}$ \\ ${ }^{1}$ Univ. Bordeaux, LaBRI, UMR 5800, PICTURA, F-33400 Talence, France \\ ${ }^{2}$ CNRS, LaBRI, UMR 5800, PICTURA, F-33400 Talence, France \\ 3 Bordeaux INP, LaBRI, UMR 5800, PICTURA, F-33600 Pessac, France \\ ${ }^{4}$ Universitat Politècnia de València, ITACA, 46022 Valencia, Spain
}

\begin{abstract}
Alzheimer's disease is the most common dementia leading to an irreversible neurodegenerative process. To date, subject revealed advanced brain structural alterations when the diagnosis is established. Therefore, an earlier diagnosis of this dementia is crucial although it is a challenging task. Recently, many studies have proposed biomarkers to perform early detection of Alzheimer's disease. Some of them have proposed methods based on inter-subject similarity while other approaches have investigated framework using intra-subject variability. In this work, we propose a novel framework combining both approaches within an efficient graph of brain structures grading. Subsequently, we demonstrate the competitive performance of the proposed method compared to stateof-the-art methods.
\end{abstract}

Keywords: Patch-based grading, Intra-subject variability, Inter-subject similarity, Alzheimer's disease classification, Mild Cognitive Impairment.

\section{Introduction}

Alzheimer's disease (AD) is the most common dementia leading to a neurodegenerative process causing mental dysfunctions. According to the world health organization, the number of patients having AD will double in 20 years. Neuroimaging studies performed on $\mathrm{AD}$ subjects revealed that brain structural alterations are advanced when diagnosis is established. Indeed, the clinical symptoms of AD is preceded by brain changes that stress the need to develop new biomarkers to detect the first stages of the disease. The development of such biomarkers can make easier the design of clinical trials and therefore accelerate the development of new therapies.

Over the past decades, the improvement of magnetic resonance imaging (MRI) has led to the development of new imaging biomarkers [2]. Many works

\footnotetext{
^ Data used in preparation of this article were obtained from the Alzheimer's Disease Neuroimaging Initiative (ADNI) database (adni.loni.usc.edu). As such, the investigators within the ADNI contributed to the design and implementation of ADNI and/or provided data but did not participate in analysis or writing of this report. A complete listing of ADNI investigators can be found at: http://adni.loni.usc.edu/wp-content/uploads/how_to_apply/ADNI_Acknowledgement_List.pdf.
} 
developed biomarkers based on inter-subject similarities to detect anatomical alterations by using group-based comparison (e.g., patients vs. normal controls). Some of them are based on regions of interest (ROI) to capture brain structural alterations at a large scale of analysis. The alterations of specific structures such as the cerebral cortex and hippocampus (HIPP) are usually captured with volume, shape, or cortical thickness (CT) measurements [17]. Other approaches proposed to study the inter-subject similarity between individuals from the same group at a voxel scale. Such methods commonly use voxel-based morphometry (VBM). VBM-based studies showed that the medial temporal lobe (MTL) is a key area to detect the first manifestations of AD [17. Recently, more advanced methods have been designed to improve computer-aided diagnosis [2]. Among them, patch-based grading (PBG) framework [3] proposed to better analyze inter-subject similarities. PBG demonstrated state-of-the-art results for $\mathrm{AD}$ diagnosis and prognosis 3 [5]13.

Beside inter-subject similarity approaches, other methods proposed to capture the correlation of brain structures alterations within subjects. Indeed, although similarity-based biomarkers provide helpful tools to detect the first signs of $\mathrm{AD}$, the structural alterations leading to cognitive decline are not homogeneous within a given subject. Such biomarkers assumed that the structural changes caused by the disease may not occur at isolated areas but in several inter-related regions. Therefore, intra-subject variability features provide relevant information. Some methods proposed to capture the relationship of spread cortical atrophy with a network-based framework [16. Other approaches estimate inter-regional correlation of brain tissues volumes [18]. Recently, convolutional neural network (CNN) have been used to capture relationship between anatomical structures volumes [11. Finally, some works showed that patch-based strategy can be used to model intra-subject brain alteration [7]12].

The main contribution of this work is the development of a novel representation based on a graph of brain structures grading (GBSG) combining intersubject pattern similarity and intra-subject variability features to better capture AD signature. First, inter-subject similarities are captured using patch-based grading framework applied over the entire brain. Second, intra-subject variabilities are modeled by a graph representation. In our experiments, we compare the performance of intra-subject variability features (i.e., the edges of our graph) with inter-subject pattern similarity features (i.e., the vertices). Moreover, we demonstrate the capability of intra-subject variability features to early detect $\mathrm{AD}$ and show that the combination of both features improves $\mathrm{AD}$ prognosis. Finally, we present competitive results of our new method compared to state-ofthe-art approaches. 


\section{Materials and Methods}

\section{$2.1 \quad$ Dataset}

Data used in this work were obtained from the Alzheimer's Disease Neuroimaging Initiative (ADNI) dataset ${ }^{1}$. We use all the baseline T1-weighted (T1w) MRI of the ADNI1 phase. This dataset includes AD patients, subjects with mild cognitive impairment (MCI) and cognitive normal (CN) subjects (see Table 1). MCI is a presymptomatic phase of AD composed of subjects who have abnormal memory dysfunctions. In our experiments we consider two groups of MCI. The first group is composed of patients having stable MCI (sMCI) and the second one is composed with patients having MCI symptoms at the baseline and converted to $\mathrm{AD}$ into the following 36 months. This group is named progressive MCI (pMCI).

Table 1. Description of the ADNI dataset used in this work.

\begin{tabular}{lcccc}
\hline Characteristic / Group & CN & sMCI & pMCI & AD \\
\hline Number of subjects & 228 & 100 & 164 & 191 \\
Ages (years) & $75.8 \pm 5.0$ & $75.3 \pm 7.2$ & $74.2 \pm 6.64$ & $75.26 \pm 7.4$ \\
Sex (M/F) & $118 / 110$ & $66 / 34$ & $97 / 67$ & $100 / 91$ \\
MMSE & $29.05 \pm 0.9$ & $27.1 \pm 2.5$ & $26.3 \pm 2.0$ & $22.8 \pm 2.9$ \\
\hline
\end{tabular}

\section{$2.2 \quad$ Preprocessing}

The data are preprocessed using the following steps: (1) denoising using a spatially adaptive non-local means filter [8], (2) inhomogeneity correction using N4 method [14], (3) low-dimensional non-linear registration to MNI152 space using ANTS software [1, (4) intensity standardization, (5) segmentation using a nonlocal label fusion [4] and (6) systematic error corrections [15]. The patch-based multi-template segmentation was performed using 35 images manually labeled by Neuromorphometrics, Inc. 5 using the brain-COLOR labeling protocol composed of 134 structures.

\subsection{Computation of patch-based grading biomarkers}

To capture alterations caused by $\mathrm{AD}$, we use the recently developed patch-based grading framework [3]. PBG framework provides at each voxel a grade between -1 and 1 related to the alteration severity. The grading value $g$ at $x_{i}$ is defined as:

$$
g_{x_{i}}=\frac{\sum_{t_{j} \in K_{i}} w\left(P_{x_{i}}, P_{t_{j}}\right) p_{t}}{\sum_{t_{j} \in K_{i}} w\left(P_{x_{i}}, P_{t_{j}}\right)}
$$

\footnotetext{
1 http://adni.Ioni.ucla.edu

5 http://Neuromorphometrics.com
} 
where $P_{x_{i}}$ and $P_{t_{j}}$ represent the patches surrounding the voxel $i$ of the test subject image $x$ and the voxel $j$ of the template image $t$, respectively. The template $t$ comes from a training library composed of $\mathrm{CN}$ subjects and $\mathrm{AD}$ patients. $p_{t}$ is the pathological status set to -1 for patches extracted from $\mathrm{AD}$ patients and to 1 for those extracted from CN subjects. $K_{i}$ is a set of the most similar $P_{t_{j}}$ patches to $P_{x_{i}}$ found in the training library. The anatomical similarity between the test subject $x$ and the training library is estimated by a weight function $w\left(P_{x_{i}}, P_{t_{j}}\right)=\exp \left(-\left\|P_{x_{i}}-P_{t_{j}}\right\|_{2}^{2} /\left(h^{2}+\epsilon\right)\right)$, where $h=\min _{t_{j}}\left\|P_{x_{i}}-P_{t_{j}}\right\|_{2}^{2}$ and $\epsilon \rightarrow 0$.

\subsection{Graph construction}

In our GBSG method, the grading process is carried out over the entire brain. Afterwards, the corresponding segmentation is used to fuse grading values and to built our graph (see Fig. 1). We define an undirected graph $G=(V, E, \Gamma, \omega)$, where $V=\left\{v_{1}, \ldots, v_{N}\right\}$ is the set of vertices for the $N$ considered brain structures and $E=V \times V$ is the set of edges. In our work, the vertices are the mean of the grading values for a given structure while the edges are based on grading distributions distances between two structures (see Fig. 1).

To this end, the probability distributions of PBG values (see Eq. 1) are estimated with a histogram $H_{v}$ for each structure $v$. The number of bins is computed with the Sturge's rule [19]. For each vertex we assign a function $\Gamma$ : $V \rightarrow \mathbb{R}$ defined as $\Gamma(v)=\mu_{H_{v}}$, where $\mu_{H_{v}}$ is the mean of $H_{v}$. For each edge we assign a weight given by the function $\omega: E \rightarrow \mathbb{R}$ defined as $\omega\left(v_{i}, v_{j}\right)=$ $\exp \left(-d\left(H_{v_{i}}, H_{v_{j}}\right)^{2} / \sigma^{2}\right)$ where $d$ is the Wasserstein distance with $L_{1}$ norm [10] that showed best performance during our experiments.

Graph representation of structure grading provides high-dimensional features (see Fig. 1). In this work we used the Elastic Net regression (EN) method that provides a sparse representation of the most discriminative edges and vertices, and thus enables to reduce the feature dimensionality by capturing the key structures and the key relationships between the different brain structures (see Fig. 2). Thus, after normalization, a concatenation of the two feature vectors is given as input of EN feature selection method.

\subsection{Details of implementation}

PBG was computed with a patch-match method 4. We used the parameters proposed in [5] for patch size and size of $K_{i}$. This results in a whole brain grading in about 10 seconds. Age effect is corrected using linear regression estimated on CN population. The EN method is computed with SLEP package [6]. Two classifiers were used to validate our method - a support vector machine (SVM) 6 . with a linear kernel and a random forest $(\mathrm{RF}){ }^{7}$. The linear SVM has a soft margin parameter, which was optimized in a range of $2^{i}$, with $i=\{-10,9, \ldots, 10\}$.

$\begin{array}{ll}7 & \text { http://www.csie.ntu.edu.tw/ }{ }^{\sim} \text { cjlin/libsvm } \\ 7 & \text { http://code.google.com/p/randomforest-matlab }\end{array}$ 
All features were normalized using z-score. In our experiments, we performed sMCI versus pMCI classification. The EN features selection and the classifiers were trained with $\mathrm{CN}$ and $\mathrm{AD}$ (see Fig. 2). As shown in [13], the use of CN and $\mathrm{AD}$ to train the feature selection method and the classifier enables to better discriminate sMCI and pMCI subjects. Moreover, it also enables to get the results without cross-validation step and more importantly to limit bias and overfitting problem. Thus, only one run was performed for the SVM and 30 runs was performed to capture the inner variability of RF. The mean accuracy (ACC), sensibility (SEN), and specificity (SPE) over these 30 iterations are provided as results (see Table 2).

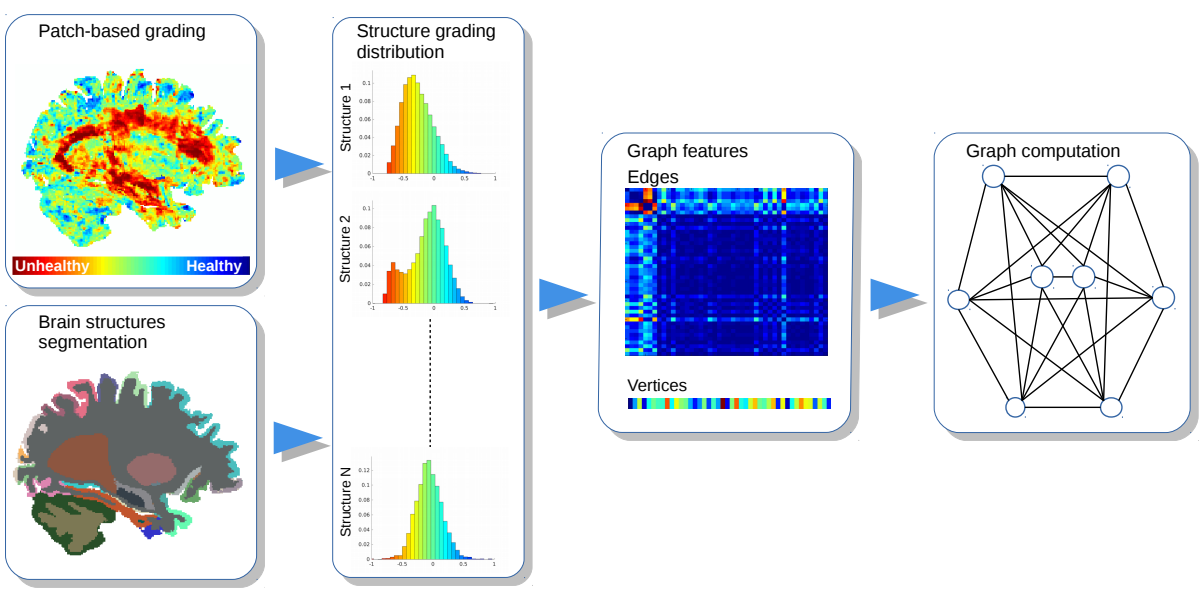

Fig. 1. Illustration of the graph construction method. From left to right, for each segmented structure an estimation of the density probability of PBG values are computed. Then, histograms are used to built our graph of brain structure grading. Thus, 134 histograms representing each segmented brain structures are estimated. Edges are the distances between structure grading distribution while vertices are the mean grading value for a given structure (see text for more details).

\section{$3 \quad$ Results and Discussions}

To investigate the results of our new GBSG method combining inter-subject pattern similarity features (i.e., vertices) and intra-subject variability features (i.e., the edges) several experiments were performed (see Table 2). The original hippocampal grading (HIPP PBG) is used as baseline [3].

First, we estimated the classification performances obtained by each feature separately using SVM. Compared to HIPP PBG, vertices showed an improvement of the specificity while the accuracy and the sensibility did not change. Therefore, additional structures selected by EN did not improve results compared 


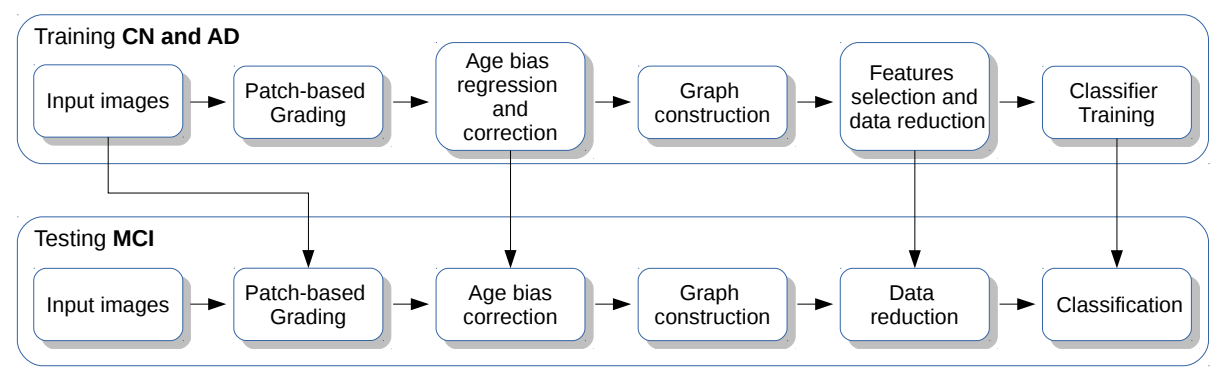

Fig. 2. Scheme of the proposed GBSG pipeline. PBG is computed using CN and AD training groups. CN group is also used to correct the bias related to age. This estimation is applied then to AD and MCI subjects. Afterwards, the graph is constructed, the feature selection is trained on $\mathrm{CN}$ and $\mathrm{AD}$ and is applied to $\mathrm{CN}, \mathrm{AD}$ and MCI. Finally the classifier is trained with $\mathrm{CN}$ and $\mathrm{AD}$.

Table 2. Classification of sMCI versus pMCI. Results obtained by inter-subject similarity features (i.e., vertices), intra-subject variability features (i.e., edges) and a combination of both. The original PBG applied on HIPP [3] is used as baseline. Results show that GBSG edge features improve the accuracy and the sensibility as compared to HIPP PBG and GBSG vertices features. Finally, GSBG provides the best results.

\begin{tabular}{lcccc}
\hline Methods & Classifier & ACC & SEN & SPE \\
\hline HIPP PBG & SVM & $71.5 \%$ & $72.5 \%$ & $70.0 \%$ \\
GBSG Vertices & SVM & $71.9 \%$ & $71.95 \%$ & $\mathbf{7 2 . 0 \%}$ \\
& RF & $70.1 \%$ & $69.6 \%$ & $71.1 \%$ \\
GBSG Edges & SVM & $74.6 \%$ & $81.7 \%$ & $63.0 \%$ \\
& RF & $73.8 \%$ & $81.3 \%$ & $61.6 \%$ \\
GBSG & SVM & $75.8 \%$ & $82.3 \%$ & $65.0 \%$ \\
& RF & $\mathbf{7 6 . 5 \%}$ & $\mathbf{8 1 . 7 \%}$ & $68.0 \%$ \\
\hline
\end{tabular}

to use HIPP only. On the other hand, the edges feature improved the accuracy and the sensibility but was less specific compared to HIPP PBG and vertices. These results indicate that relevant information is encoded within GBSG edges.

Second, we evaluated the performance of combining vertex and edge features. GBSG provided the best results in terms of accuracy and sensibility. Moreover it improved the specificity compared to the intra-subject variability features. Finally, we compared SVM and RF classifiers to study the stability of our framework. The results obtained with both classifier showed the same tendency. The $\mathrm{RF}$ provided the best results with $76.5 \%$ of accuracy.

These results obtained with two different classification methods demonstrate the complementarity of inter-subject similarity and intra-subject variability features. Indeed, both information - level of structure degradations and global pattern of key structure modifications - are relevant.

Afterwards, we compared our GBSG method using RF classifier with stateof-the-art methods on similar ADNI1 datasets. First, we included methods modeling inter-subject variability based on PBG within HIPP [3, VBM [9] and an 
advanced PBG (aPBG) estimated over the entire brain [13]. Second, we included methods capturing intra-subject variability based on last deep learning framework [11], multiple instance learning (MIL) [12] and integrative network of cortical thickness abnormality (ICT) [16. We applied our GBSG on two definitions of sMCI/pMCI populations as defined in [9] and [13] to perform a fair comparison. Results of this comparison are presented in Table 3. This comparison shows that best methods based on intra-subject or inter-subject obtained similar accuracy around $75 \%$ while our GBSG combining both reached $76.5 \%$ of accuracy. Compared to VBM on the same dataset [9, our GBSG improved accuracy by 1.8 percent point. However, compared to aPBG [13] GBSG provided similar results on the same dataset. Finally, compared to the CNN-based method proposed in [11] our method obtained competitive performances. These results highlight the efficiency of combining intra-subject and inter-subject features.

Table 3. Comparison of the proposed method with state-of-the-art approaches. These results show the competitive performance of our new GBSG method that obtains the best accuracy on both definitions of sMCI/pMCI populations.

\begin{tabular}{lcccccc}
\hline Method & sMCI/pMCI & Area & Feature & ACC & SEN & SPE \\
\hline PBG [3] & $238 / 167$ & HIPP & Inter & $71.0 \%$ & $70.0 \%$ & $71.0 \%$ \\
VBM [9] & $100 / 164$ & Brain & Inter & $74.7 \%$ & $88.8 \%$ & $51.59 \%$ \\
aPBG [13] & $129 / 171$ & Brain & Inter & $75.0 \%$ & - & - \\
\hline ICT [16] & $111 / 89$ & Cortex & Intra & $75.0 \%$ & $63.5 \%$ & $84.4 \%$ \\
MIL [1] & $238 / 167$ & MTL & Intra & $72.0 \%$ & $69.0 \%$ & $74.0 \%$ \\
CNN [11] & $226 / 167$ & GM & Intra & $74.8 \%$ & $70.9 \%$ & $78.8 \%$ \\
\hline GBSG & $129 / 171$ & Brain & Inter + Intra & $\mathbf{7 5 . 2 \%}$ & $80.0 \%$ & $68.7 \%$ \\
& $100 / 164$ & Brain & Inter + Intra & $\mathbf{7 6 . 5 \%}$ & $81.7 \%$ & $68.0 \%$ \\
\hline
\end{tabular}

\section{Conclusions}

In this paper, we proposed a novel framework based on a promising graph of brain structures grading. Our new method combines inter-subject pattern similarities and intra-subject variabilities to better detect $\mathrm{AD}$ alterations. The pattern similarity is estimated with a patch-based grading strategy, while the intra-subject variability between structures grading is based on graph modeling. Our experiments showed the complementarity of both information. Finally, we demonstrated that our method obtains competitive performance compared to the most advanced methods.

Acknowledgement This study has been carried out with financial support from the French State, managed by the French National Research Agency (ANR) in the frame of the Investments for the future Program IdEx Bordeaux (HL-MRI ANR-10-IDEX-03-02), Cluster of excellence CPU and TRAIL (HR-DTI ANR10-LABX-57). 


\section{References}

1. Avants et al. A reproducible evaluation of ANTs similarity metric performance in brain image registration. Neuroimage, 54(3):2033-2044, 2011.

2. Bron et al. Standardized evaluation of algorithms for computer-aided diagnosis of dementia based on structural MRI: The CADDementia challenge. NeuroImage, 111:562-579, 2015.

3. Coupé et al. Scoring by nonlocal image patch estimator for early detection of Alzheimer's disease. NeuroImage: clinical, 1(1):141-152, 2012.

4. Giraud et al. An optimized patchmatch for multi-scale and multi-feature label fusion. NeuroImage, 124:770-782, 2016.

5. Hett et al. Adaptive fusion of texture-based grading: Application to Alzheimer's disease detection. In International Workshop on Patch-based Techniques in Medical Imaging, pages 82-89. Springer, 2017.

6. Liu et al. Slep: Sparse learning with efficient projections. Arizona State University, $6(491): 7,2009$.

7. Liu et al. Hierarchical fusion of features and classifier decisions for Alzheimer's disease diagnosis. Human brain mapping, 35(4):1305-1319, 2014.

8. Manjón et al. Adaptive non-local means denoising of MR images with spatially varying noise levels. Journal of Magnetic Resonance Imaging, 31(1):192-203, 2010.

9. Moradi et al. Machine learning framework for early MRI-based Alzheimer's conversion prediction in MCI subjects. Neuroimage, 104:398-412, 2015.

10. Rubner et al. The earth mover's distance as a metric for image retrieval. International journal of computer vision, 40(2):99-121, 2000.

11. Suk et al. Deep ensemble learning of sparse regression models for brain disease diagnosis. Medical Image Analysis, 37:101-113, 2017.

12. Tong et al. Multiple instance learning for classification of dementia in brain MRI. Medical Image Analysis, 18(5):808-818, 2014.

13. Tong et al. A novel grading biomarker for the prediction of conversion from mild cognitive impairment to Alzheimer's disease. IEEE Transactions on Biomedical Engineering, 64(1):155-165, 2017.

14. Tustison et al. N4ITK: improved N3 bias correction. IEEE Transactions on Medical Imaging, 29(6):1310-1320, 2010.

15. Wang et al. A learning-based wrapper method to correct systematic errors in automatic image segmentation: consistently improved performance in hippocampus, cortex and brain segmentation. NeuroImage, 55(3):968-985, 2011.

16. Wee et al. Prediction of Alzheimer's disease and mild cognitive impairment using cortical morphological patterns. Human brain mapping, 34(12):3411-3425, 2013.

17. Wolz et al. Multi-method analysis of MRI images in early diagnostics of Alzheimer's disease. PloS one, 6(10):e25446, 2011.

18. Zhou et al. Hierarchical anatomical brain networks for MCI prediction: revisiting volumetric measures. PloS one, 6(7):e21935, 2011.

19. Sturges. The choice of a class interval. Journal of the american statistical association, 21(153):65-66, 1926. 